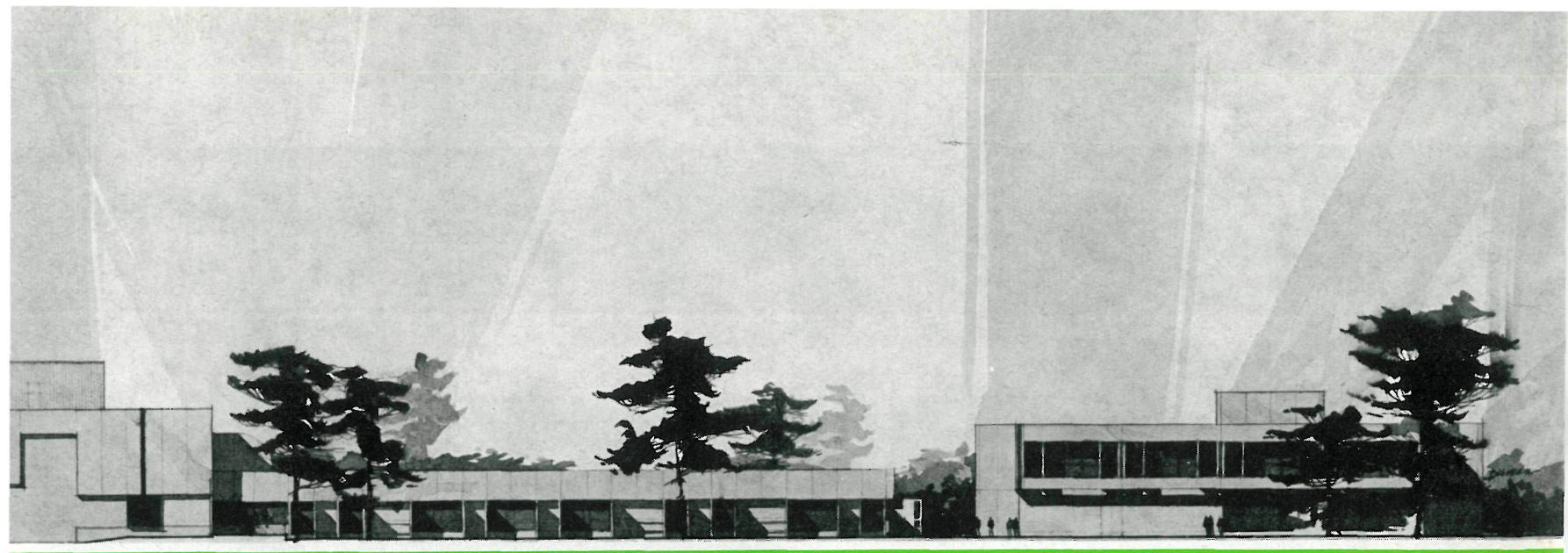

\title{
Delta Greenbriar
} Atlanta EE. UU.

HEERY \& HEERY, arquitectos e ingenieros

$131-104$

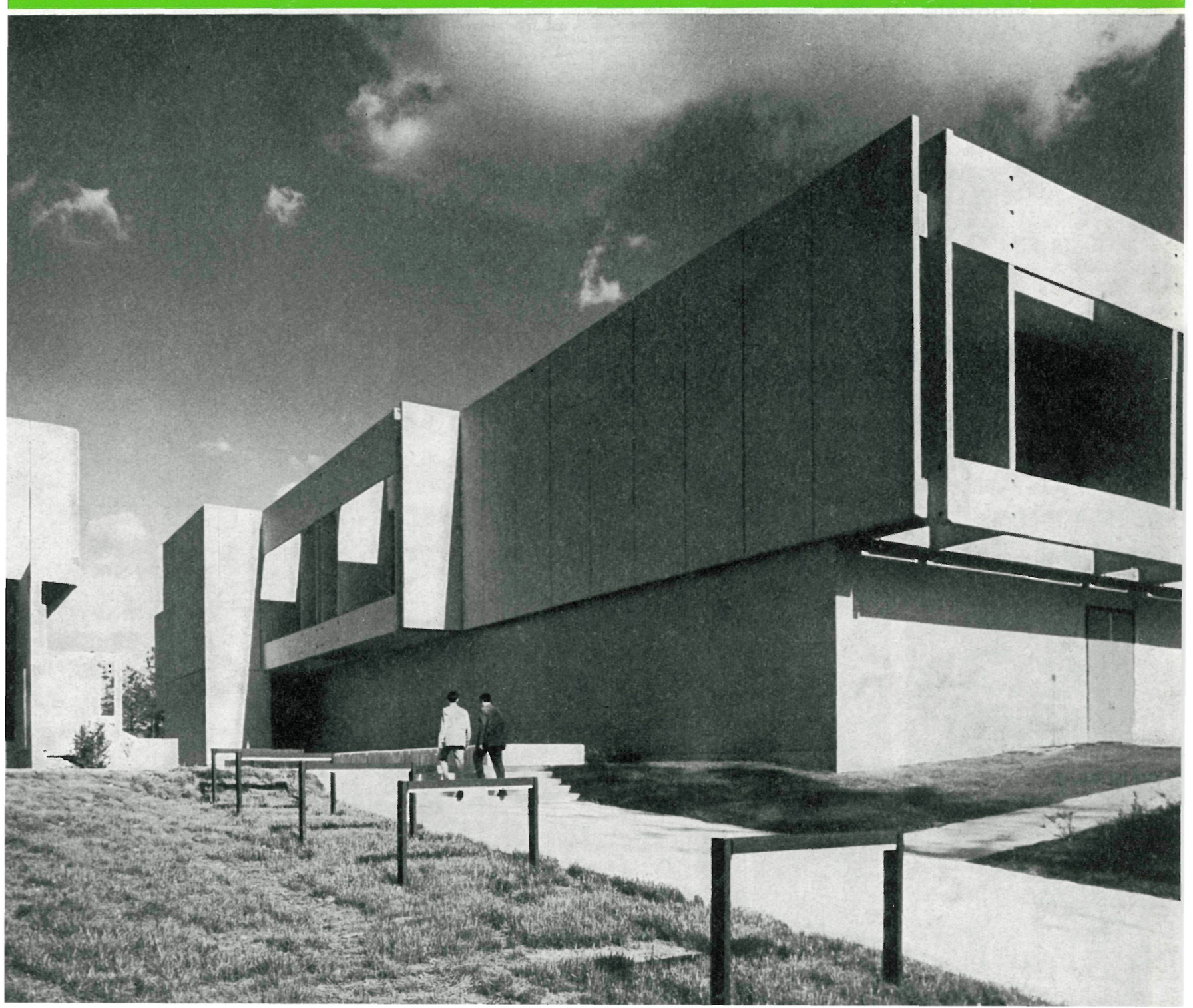




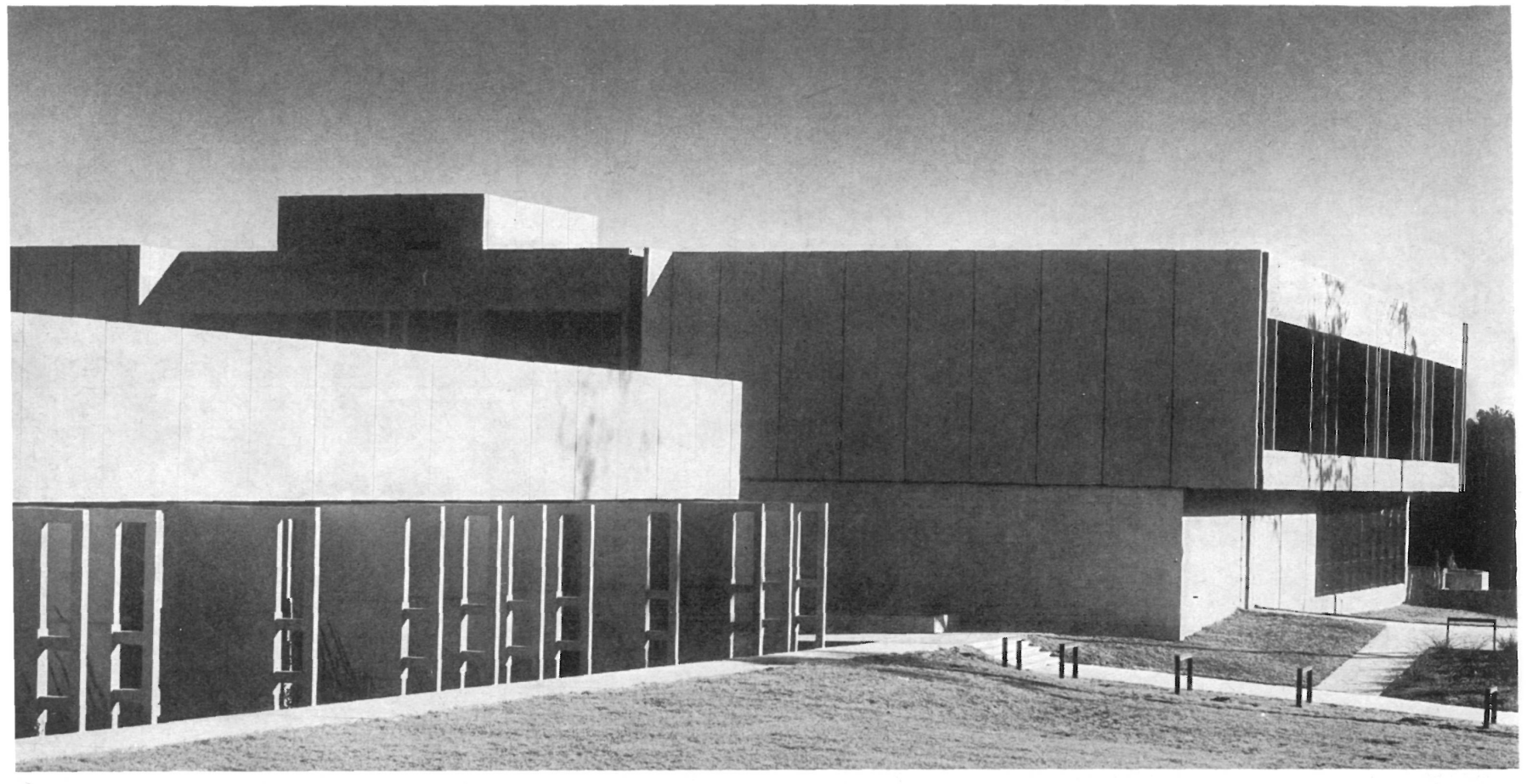

Sinopsis El “Delta Greenbriar" es un centro corporativo para la compañía de líneas aéreas Delta. El "Delta Greenbriar" es un centro corporativo para la compañía de líneas aéreas Delta.
El complejo está constituido por cuatro edificios, que alojan: 1) las instalaciones para la
simulación de vuelo; 2) la base de entrenamiento aéreo; 3) el centro de control de vuelo
y comunicaciones; 4) el departamento de reservas y venta de billetes. Constan de una o dos y comunicaciones; 4) el departamento de reservas y venta de billetes. Constan de una o dos plantas, pudiéndose ampliar los más bajos tanto horizontal como verticalmente, mientras que los otros sólo pueden aumentarse en sentido horizontal.

El conjunto se ha diseñado como un campus universítario, con patios, ajardinamiento interior y corredores exteriores que, además de enlazar los distintos edificios entre sí, ponen en

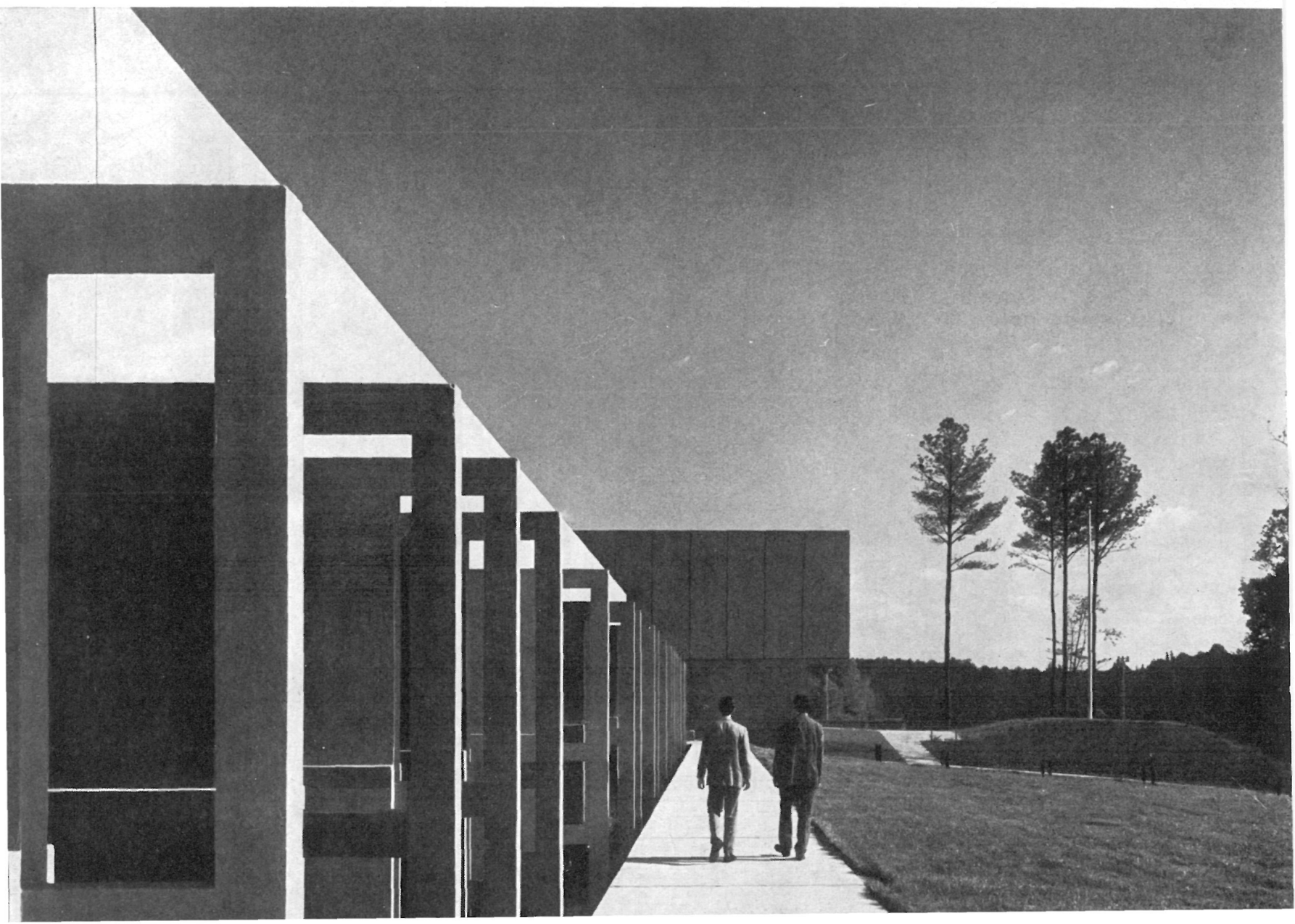

(c) Consejo Superior de Investigaciones Científicas Licencia Creative Commons 3.0 España (by-nc) 
El complejo Delta Greenbriar, constituido por cuatro edificios, forma parte de un gran centro corporativo para la línea aérea Delta, con base en Atlanta.

Las instalaciones tuvieron que ser diseñadas y construidas en sólo doce meses y medio, para permitir la entrega, a la compañía Delta, del primero de sus simuladores de vuelo. Para conseguir esto, los arquitectos utilizaron un esquema basado en fases sucesivas, diseñando y estudiando las propuestas iniciales para los sistemas de estructura y de HVAC del simulador de vuelo. Los estudios de las propuestas estructurales se realizaron con anterioridad a la terminación del diseño del resto del conjunto. Este esquema de trabajo se combinó con una técnica de diseño y dirección de obra, denominada de control de tiempos/costes, la cual permitió, desde el primer momento, estimar, con mucha exactitud, tanto la duración de las obras como el coste final.

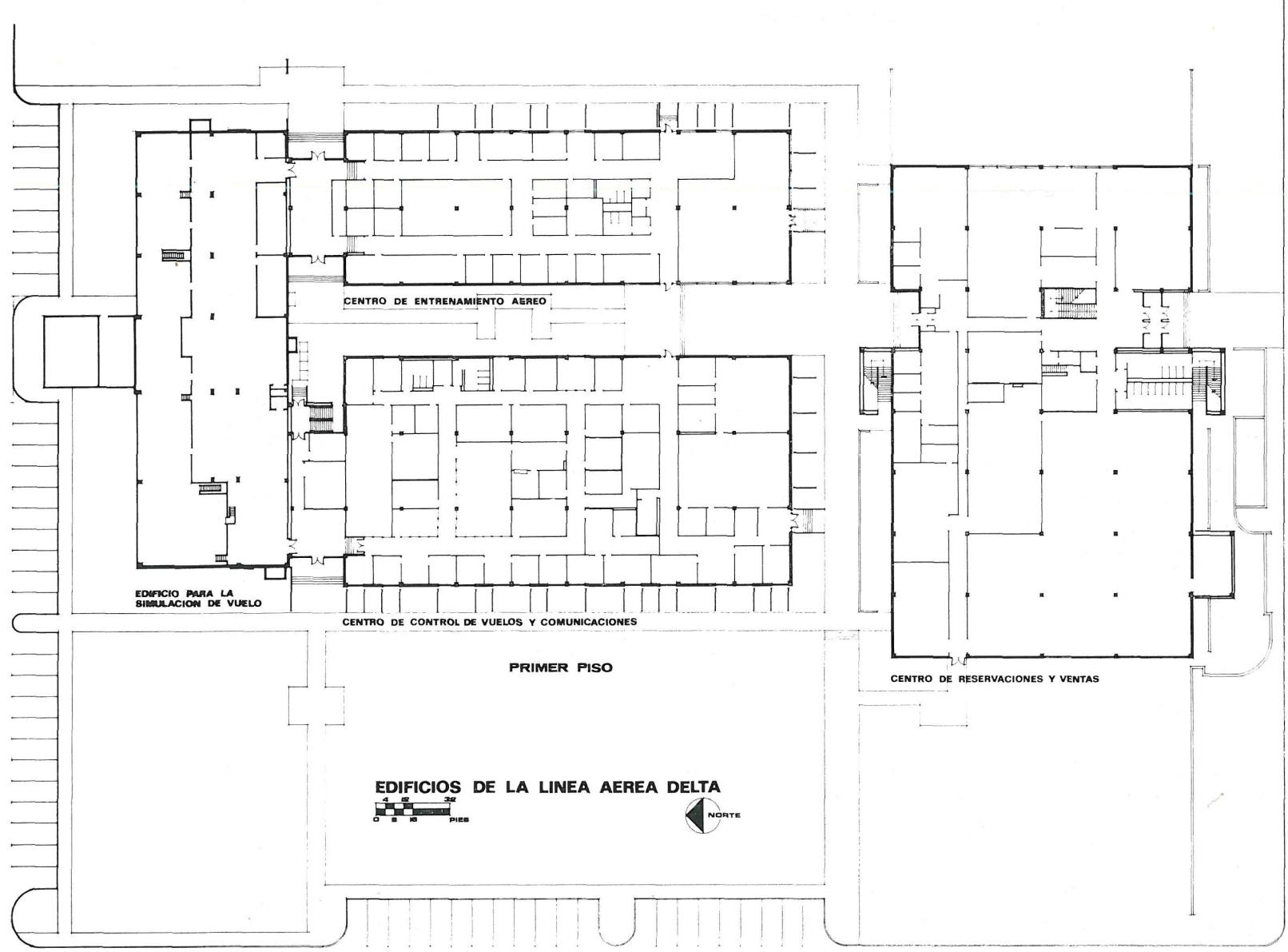

planta baja 


\section{planta alta}

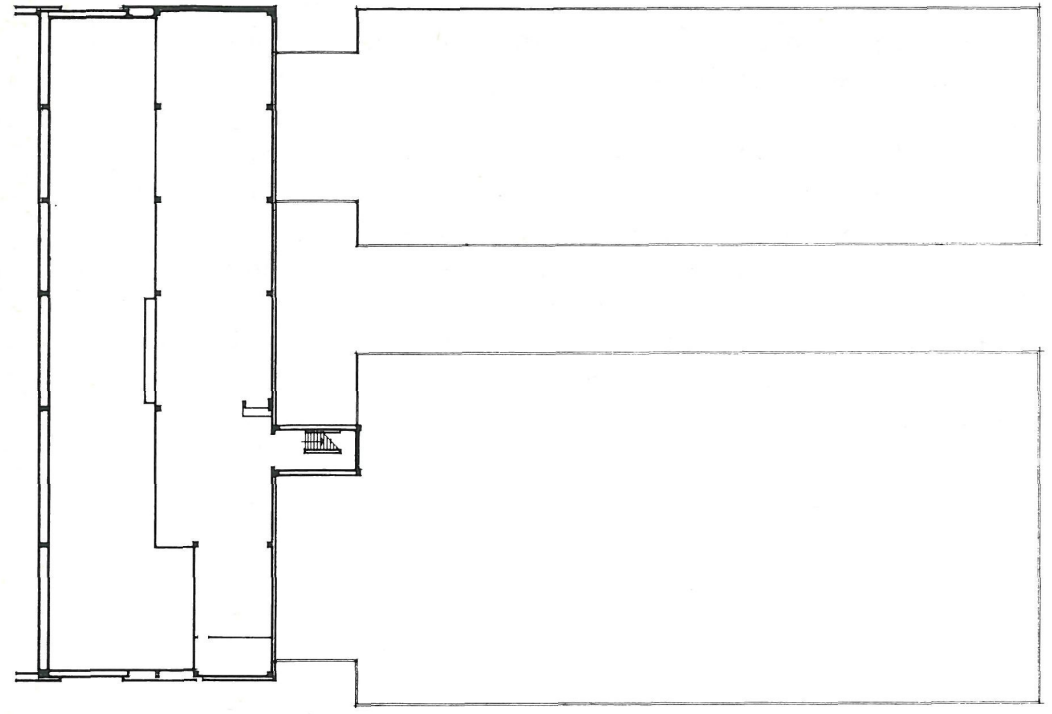

SEGUNDO PISO

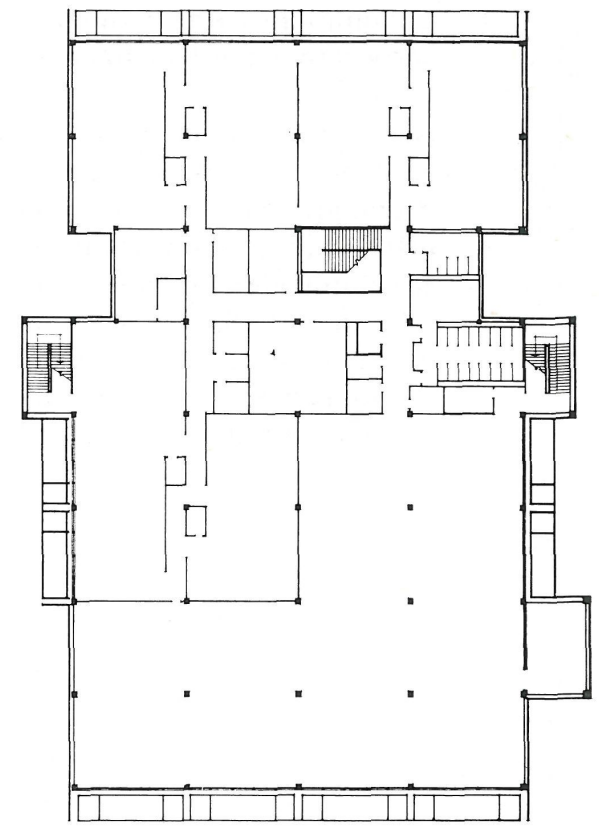

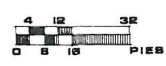

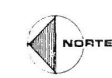

El complejo, asentado sobre una superficie de $10.700 \mathrm{~m}^{2}$, lo forman los cuatro edificios siguientes: al norte de la parcela, el de las instalaciones para el principal simulador de vuelo de la casa Delta, de dos plantas; en la parte central y colocados al este y al oeste, respectivamente, el centro de entrenamiento aéreo para pilotos, y el centro de control de vuelo, comunicaciones y meteorología, ambos de una sola planta; y por último, en la zona sur, el departamento central de ventas y reservas de billetes, de dos plantas.

Las dos construcciones centrales están unidas directamente al edificio para la simulación de vuelo mediante dos distribuidores cubiertos, estableciéndose el enlace entre todos los edificios por medio de un patio interior abierto y los corredores exteriores que los circundan.

Los dos edificios de una sola planta se diseñaron para que, en caso necesario, pudieran crecer tanto vertical como horizontalmente, mientras que los de dos plantas fueron planeados para una posible ampliación únicamente en horizontal.

El terreno no construido, que incluye un pequeño lago natural, se trató como un campus universitario con patios, corredores y zonas ajardinadas, para que los empleados -la mayoría de los cuales desarrollan trabajos altamente especializados y de mucha tensiónpuedan disfrutar de un ambiente tranquilo y apacible desde sus puestos de trabajo. Esto era especialmente interesante para una organización cuya jornada de trabajo dura las 24 horas de cada día de la semana, con numerosos empleados en cualquier momento del día o de la noche. 
Se puede decir, por último, del Delta Greenbriar, que es un nuevo tipo de centro corporativo, ecológicamente afín con su emplazamiento, gracias a la amplitud del paisaje exterior y del ajardinamiento interior, que permiten una estrecha relación entre los espacios de oficinas y la atmósfera del parque que las envuelve.

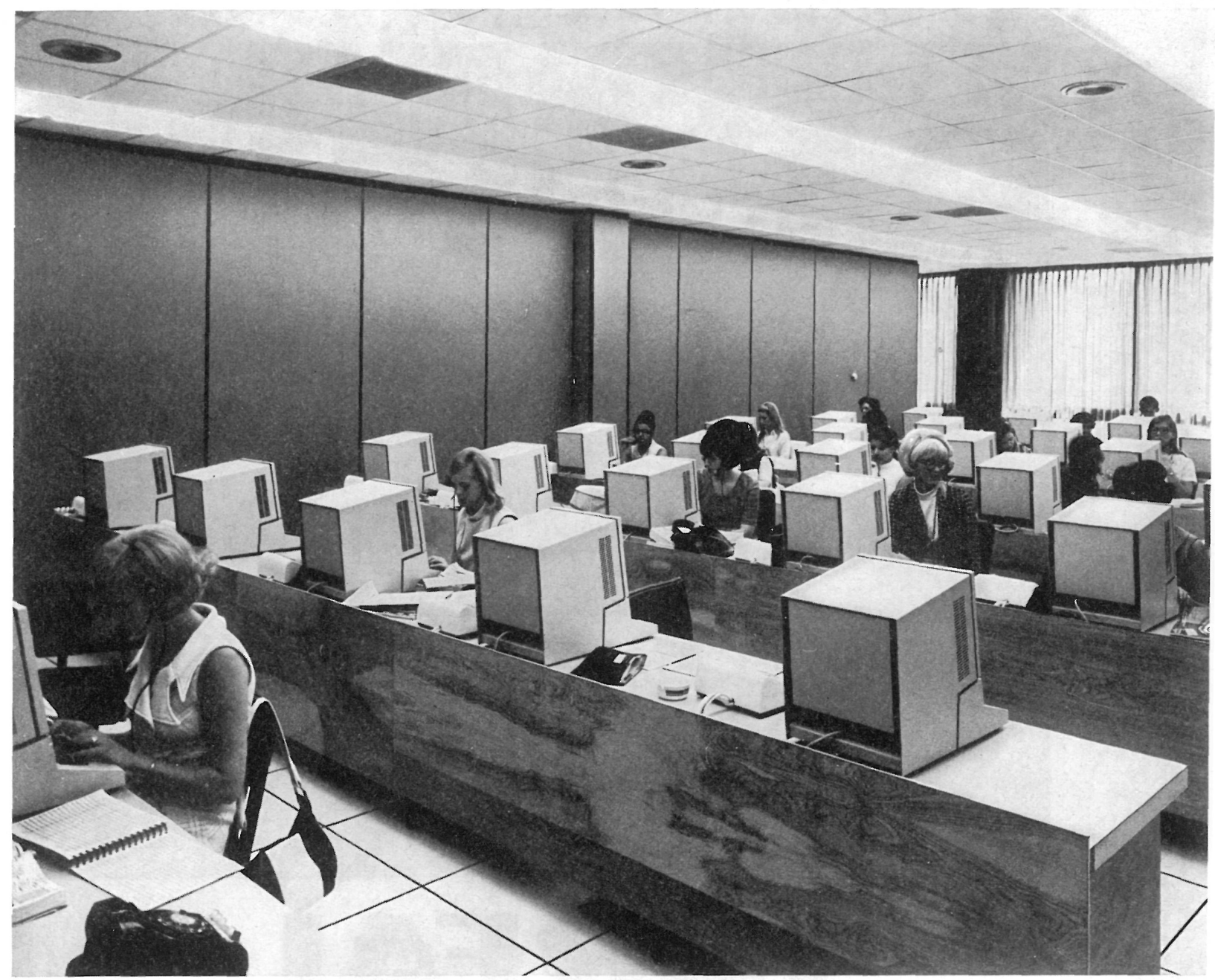

\section{résumé}

Delta Greenbriar - Atlanta.

Etats-Unis d'Amérique

Heery \& Heery, architectes et ingénieurs

Le "Delta Greenbriar" est un centre corporatif pour la compagnie des lignes aériennes Delta.

L'ensemble comprend quatre bâtiments, qui abritent: 1) les installations pour la simula tion de vol; 2) la base d'entraînement aérien; 3) le centre de contrôle de vol et de communications; 4) le département de réservations et de vente de billets. Ils se plus bas pouvant etre agrandis tant horizontalement que verticalement, tandis que les autres ne peuvent être agrandis qu'en sens horizontal.

L'ensemble a été conçu comme un "cam pus" universitaire, avec des patios, des es paces verts interieurs et des corridors exterieurs qui, en plus de relier les differents bâtiments entre eux, etablissent une étroite

\section{summary}

Delta Greenbriar - Atlanta. United States

Heery \& Heery, architects and engineers "Delta Greenbriar" is a corporate centre for the Delta air line company.

The complex comprises four buildings containing: 1) the installations for flight simucontrol 2) the air training base; 3) the tions; 4) the department for tickets and reservations. They consist of one of two storeys. The lower ones can be extended both horizontally as well as vertically in horizontal direction.

The unit has been designed like a university campus, with patios, inner gardens an exterior corridors which apart from connecting the various bulldings with each othe also provide a close contact

\section{zusammenfassung}

Delta Greenbriar - Atlanta - U.S.A

Heery \& Heery, Architekten und Ingenieure

"Delta Greenbriarn ist ein korporatives Zentrum für die Delta Fluggesellschaft.

Das Komplex besteht aus vier Gebäuden, die folgendes umfassen: 1] die Flugsimulationsanlagen; 2) die Flugtrainingsbase; 3 ) das Kontrollzentrum für Flug und Kommunikationen; 4) die Reserve- und KartenabteEtgan. Die unteren können horizontal und vertikal ausgebaut werden, während die übrigen nur in horizontaler Richtung erweitert werden können.

Der Block ist wie eine Universitätsanlage geplant worden, mit Patios, inneren Gärten und Korridoren im Freien, die sowohl die denchiedenen Gebaude takt mit dem Park 